\title{
Microbiological profile and multidrug resistant bacteria in pneumonia
}

\author{
Adriana Slavcovici ${ }^{1 *}$, Cristina Cismaru', Mihaela Sabou', Mirela Flonta², Natalia Hagău ${ }^{3}$ \\ From The 9th Edition of the Scientific Days of the National Institute for Infectious Diseases Prof Dr Matei Bals \\ Bucharest, Romania. 23-25 October 2013
}

\section{Background}

Currently accepted classifications of pneumonia include community acquired pneumonia (CAP), healthcareassociated pneumonia (HCAP), hospital-acquired pneumonia (HAP), and ventilator-associated pneumonia (VAP). Previous studies have shown a high rate of patients with HCAP or HAP caused by multidrug-resistant bacteria (MDR). Our aim was to determine the differences in etiology and antibiotic-resistant bacteria between CAP, HCAP and HAP groups.

\section{Methods}

We performed a retrospective study in which we included patients over 18 years old, with culture-positive pneumonia, between 2008 and 2011, from 3 clinical hospitals. The patients were classified as having CAP, HCAP or HAP. We recorded the bacteriologic results for the following samples: blood culture, sputum, bronchial aspirate, bronchoalveolar lavage. The statistical analysis was carried out using Graph Pad Prism 5.

\section{Results}

A total of 340 patients were recorded (160 with CAP, 39 with HCAP and 141 with HAP). Streptococcus pneumoniae and Haemophilus influenzae were seen more frequently in CAP $(33 \%, 14.4 \%)$ than in HCAP or HAP (1.4\%, 0\%; $\mathrm{p}<0.0001)$. The most common pathogens in HAP were MRSA (27.7\%), Acinetobacter spp. (26.2\%), Klebsiella pneumoniae (19\%). Compared to the HCAP, Acinetobacter spp. and MRSA were significantly associated with HAP (OR 54.8, $\mathrm{p}<0.0001$, LR 3.9). Compared to the HAP $P$ aeruginosa and $E$ coli were more common in HCAP (36\%, p 0.04, OR 2; respectively $23 \%$, p 0.001 ,
OR 7). Among Gram-negative bacilli, resistance to ceftazidime was higher $(86 \%)$ and significantly associated with HAP (OR 14.3, p<0.0001, LR 2.7). The extended-spectrum $\beta$-lactamase-producing Enterobacteriaceae, were more frequent in HAP (67.7\%). However, no differences were found regarding ESBL producing Enterobacteriaceae between HAP and HCAP ( $\mathrm{p}=0.06)$. MDR bacteria, including MRSA and gram-negative bacilli, were identified more frequently in HAP versus HCAP (78.7\% vs. $12.8 \%$; $<<0.0001$, OR 25, LR 6).

\section{Conclusion}

$S$ pneumoniae was the main causative pathogen in CAP. Despite the higher frequency of $P$ aeruginosa in the HCAP group, the MDR bacteria were associated with the HAP group. Our results reveal important differences between HAP and HCAP concerning the etiology and antibiotic resistance patterns.

\section{Authors' details}

${ }^{1}$ Department of Infectious Diseases, Iuliu Hațieganu University of Medicine and Pharmacy, Clinical Hospital of Infectious Diseases, Cluj-Napoca, Romania. ${ }^{2}$ Clinical Laboratory, Clinical Hospital of Infectious Diseases, Cluj-Napoca, Romania. ${ }^{3}$ Department of Anesthesiology and Intensive Therapy, Iuliu Hațieganu University of Medicine and Pharmacy, University Emergency County Hospital of Cluj, Cluj-Napoca, Romania.

Published: 16 December 2013

doi:10.1186/1471-2334-13-S1-013

Cite this article as: Slavcovici et al:: Microbiological profile and multidrug resistant bacteria in pneumonia. BMC Infectious Diseases 2013 13(Suppl 1):013.

\footnotetext{
* Correspondence: aslavcovici@yahoo.com

'Department of Infectious Diseases, Iuliu Hațieganu University of Medicine and Pharmacy, Clinical Hospital of Infectious Diseases, Cluj-Napoca, Romania Full list of author information is available at the end of the article
} 